\title{
Detailed characterization of stellar high energy (FUV/EUV/X-ray) radiation fields during protoplanetary system formation
}

\author{
Alexander Brown ${ }^{1}$ \\ ${ }^{1}$ Center for Astrophysics and Space Astronomy, University of Colorado, Boulder, CO \\ 80309-0389, USA \\ email: Alexander.Brown@colorado.edu
}

\begin{abstract}
Young stars undergoing the conversion of pre-main-sequence circumstellar disks into protoplanetary systems are strong sources of high energy (FUV/EUV/X-ray) radiation that controls the physical and chemical processes in their circumstellar environment out to hundreds of AU from the star. The high energy radiation resulting from magnetic activity and accretion onto the central star controls the thermal structure of disks, the formation process of planetesimals, and the photoexcitation and photoionization of protoplanets and young planetary atmospheres. Modeling of the dust and gas evolution requires an accurate understanding of the local radiation field throughout the ultraviolet (UV) and X-ray spectral regions, even those parts of the spectrum that are impossible to observe from Earth.

Our current research efforts are directed towards developing a better understanding of UV (using HST and FUSE) and X-ray (using Chandra, XMM-Newton, and Swift) stellar activity and the resulting radiation fields during pre-main-sequence evolution from ages of a few to several hundred million years. These studies include extensive UV and X-ray spectral sampling of individual stars in nearby star formation regions and the various moving groups of the Local Association, including our HST Cycle 17 Large Project (GO-11616), which is using 111 HST orbits to observe $32 \mathrm{~T}$ Tauri stars with the COS UV spectrograph. Most young stars are well over $100 \mathrm{pc}$ from the Sun and are consequently hard to observe in the UV and X-ray regions at even moderate spectral resolution. However, members of the Local Association, whose ages range from $7 \mathrm{Myr}$ to a few hundred Myr, surround the Sun at distances of $50 \mathrm{pc}$ or less and permit the detailed study of the later stages of the early evolution of stellar activity when gas giant and terrestrial protoplanets are forming. We illustrate our methodology using the $12 \mathrm{Myr}$ old early-M dwarf AU Mic, which possesses a striking dust debris disk, as an example.
\end{abstract}

Keywords. stars: pre-main-sequence, stars: activity, planetary systems: formation, planetary systems: protoplanetary disks

\section{Introduction}

Protoplanetary systems are highly complex with a magnetically-active central star surrounded by an accretion disk and with the interaction between them driving strong directional outflows. The overall kinematics and energy of the system is controlled by conservation of angular momentum, but with accretion onto star and the outflow geometry constrained by magnetic fields. The physics and chemistry of the circumstellar environment for tens - hundreds of AU around the star are controlled by the stellar radiation field. Pre-main-sequence (PMS) circumstellar disks would be very cold (and uninteresting!) if only subject to stellar photospheric and interstellar radiation fields. However, stellar high energy (X-ray/UV) photons significantly alter the thermal structure of the disk with X-ray and far-UV (FUV — between the H Lyman limit at $912 \AA$ and 

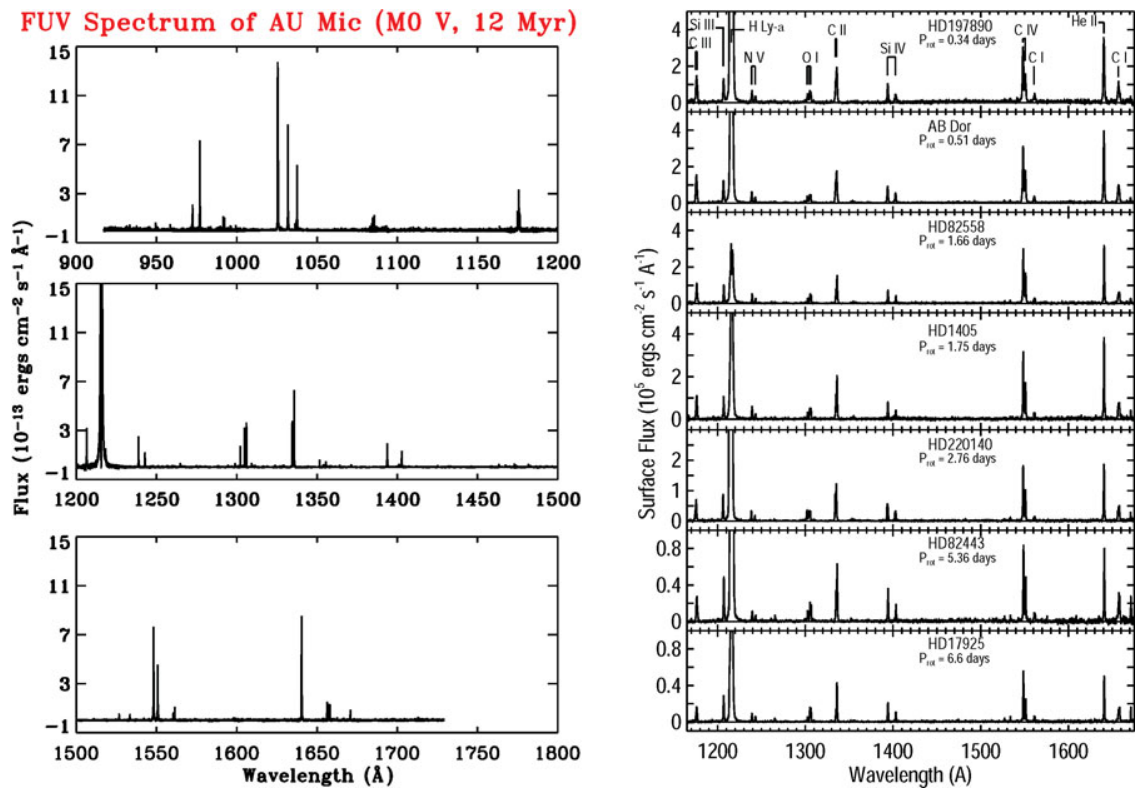

Figure 1. FUV spectra of young active PMS dwarf stars: (left) HST-STIS and FUSE spectra of the $12 \mathrm{Myr}$ old M dwarf AU Mic; (right) HST-GHRS G140L FUV spectra of a sample of early-K Local Association dwarfs - the stars are ordered by decreasing rotation period rather than by age. Flux in these FUV energy distributions is concentrated in a few strong emission lines and is thus a highly selective photoexcitation source.

$\sim 2000 \AA$ ) photons penetrating to different depths in the disk and this produces a complex layered thermal structure and regions with different chemical reaction environments (for reviews see Dullemond et al. 2007 and Bergin 2009). Over time, circumstellar disks around young, low mass stars transform much of the dust and gas in their inner regions into planetesimals or larger solid bodies. The evolutionary trend is from gas-dominated circumstellar physics to a solid-dominated structure where the gas component exists as icy surfaces on planetesimals and larger bodies. In the latter case gravitational interactions control the evolution (see e.g. Wyatt 2008; Blum \& Wurm 2008), but the radiation field controls the icy surface chemistry, both organic and inorganic.

\section{Why does the Stellar Ultraviolet and X-ray Radiation Field Matter?}

Young PMS stars are strong UV and X-ray emitters due to both strong stellar magnetic activity and the high temperature plasma generated within the accretion shock where magnetically-channeled matter impacts the star (Feigelson \& Montmerle 1999). These high energy photons have a profound effect on the conditions around the star and play an important role in photoexciting atoms, molecules and dust grains in both the accretion disk (Glassgold, Najita, \& Igea 2004) and lower density circumstellar gas. Stellar X-ray and extreme-UV (EUV) photons are the major ionization source for much of the disk (Alexander, Clarke, \& Pringle 2006) with the very hardest X-rays perhaps penetrating right to the cold disk midplane. The high energy photons drive an acceleration of disk evolution through photoevaporation (Gorti \& Hollenbach 2009; Owen et al. 2009). 


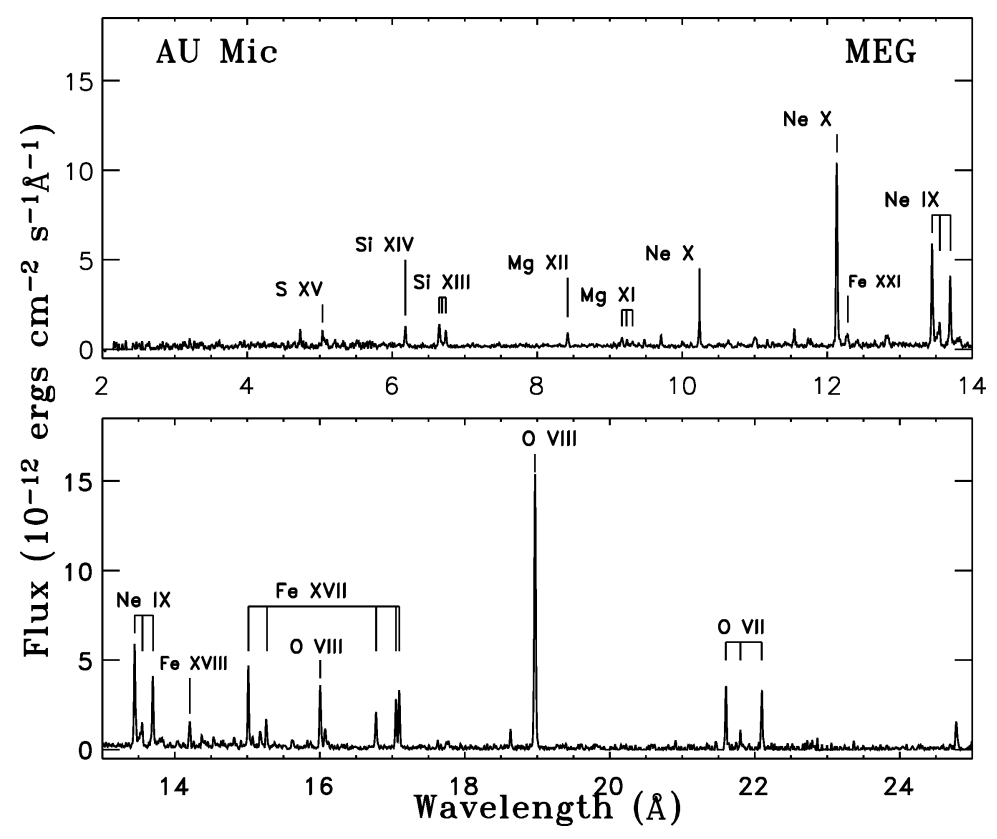

Figure 2. X-ray high resolution transmission grating spectrum of AU Mic (M0 V) as observed with the Chandra HETGS MEG spectrograph. The spectrum is dominated by strong coronal emission lines formed at temperatures between $10^{6}$ and a few $10^{7} \mathrm{~K}$. A detectable X-ray continuum is present but comparatively weak.

The stellar radiation field is the primary driver of the physical conditions and chemical processes in the gas and dust surrounding the star. These effects are important for a wide variety of situations ranging from circumstellar disks, to accreting planetesimals, and to protoplanetary atmospheres. This UV/X-ray radiation controls:

- Photoexcitation of molecules and atoms.

- Photoionization of atoms and photodissociation of molecules.

- Drives more complicated chemistry in the gas phase and on dust grain surfaces, leading potentially all the way to complex organic molecules.

- Desorption of molecules from grain surfaces.

- Photoevaporation of material from disk surface layers.

- An increase in efficiency of magnetic coupling to disk material.

Information on the full spectral energy distribution of the central star is needed for accurate modeling of the influence of the radiation field, because photons with different energies have different effects on the circumstellar environment. Strong emission lines are present throughout the whole X-ray/EUV/FUV spectral range. For young active stars the flux in the FUV is very similar to that in the $\mathrm{X}$-ray region.

FUV emission originates from upper chromospheric and transition region plasma (i.e. temperatures from just under $10^{4} \mathrm{~K}$ to a few $10^{5} \mathrm{~K}$ ), while X-rays originate from corona plasma at a few to tens MK. The FUV region dominated by emission lines with only weak continuum. Fig. 1 shows the FUV spectrum of a young M dwarf AU Mic from the Lyman limit through much of the FUV — the emission line luminosities observed for this and all other young stars are $~ 1000$ times stronger than those seen on the Sun. The other panel of Fig. 1 shows the spectra of a sample of Local Association (see below) dwarfs with similar masses but different ages and rotational periods - the strengths of 


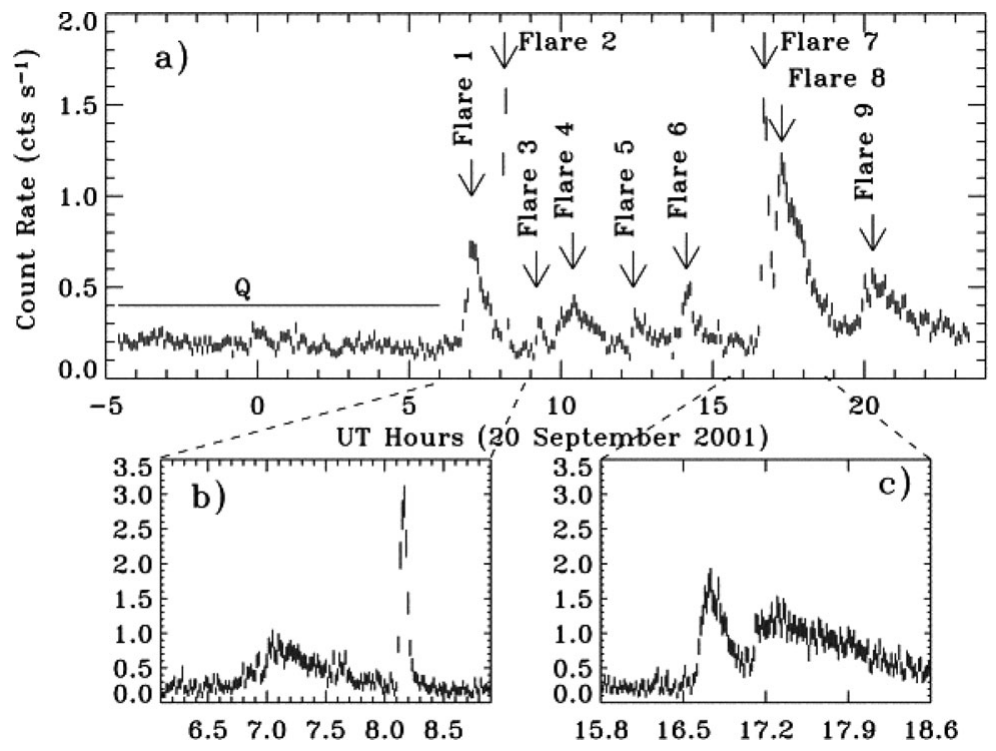

Figure 3. X-ray flaring on the $300 \mathrm{Myr}$ old M dwarf EV Lac. Light curve of Chandra first order MEG count rate during a 100 kilosecond observation on 2001 Sep 19-20 from Osten et al. (2005). The ubiquitousness of frequent factor-of-a-few flare enhancements on timescales of a few hours is well illustrated.

the emission lines, and hence the level of stellar activity, depends on the rotational period (faster rotators are more active) rather than the age.

As is immediately obvious, this radiation field is a highly-specific excitation source with most of the flux restricted to narrow energy ranges (i.e. in the emission lines). In addition the excitation routes are equally specific - only when the two match is an excitation process strongly driven. The X-ray region is also mostly dominated by emission lines but a weak continuum is clearly present (see Fig. 2). Unlike the situation for the Sun where the full spectrum from X-rays through the EUV, FUV and NUV can be observed by Earth-orbiting satellites, for even the nearest stars much of the EUV spectrum is lost to interstellar absorption. The EUV is a vital component of the radiation field that must be known for accurate modeling as it plays a vital role in hydrogen ionization and photoevaporation. However, by observing a range of UV and X-ray emission lines that sample the transition region and coronal temperature distribution the full spectral energy distribution can be modeled, because the EUV is filled by emission lines that form at upper-transition-region and lower-coronal temperatures.

\section{Variability Due to Stellar Flares}

Low mass PMS stars are undoubtedly variable X-ray and UV sources with contributions from both flaring due to magnetic reconnection and to uneven activity distributions across the stellar surface, which then modulate the observed flux as the star rotates. From the XEST survey (Güdel et al. 2007) roughly half the stars showed measurable X-ray variability during observations that lasted typically 30 kiloseconds and with half of these showing obvious flares (Stelzer et al. 2007). Large flares occurred on individual stars every ten days or so. Flares continue to be common on young stars for hundreds of Myr after accretion ceases and factor of a few flare enhancements are typically seen on few hour time-scales. Flares can exhibit a wide range of energies and time-scales — this is 
well illustrated in Fig. 3 which shows the coronal emission from the 300 Myr old M dwarf EV Lac as observed over a single day (Osten et al. (2005)). Flare outbursts are complex with each individual flares disturbing the coronal magnetic topology and triggering further flares.

A hierarchy of flaring is seen with ever larger flares being seen less frequently. Vast long-duration multi-day flare outbursts have been observed, such as the 2 day (15x quiescent at peak) flare outburst seen by Cully et al. (1993) on AU Mic with the EUVE satellite. The very largest superflares have been very difficult to observe because they occur at frequencies of $\sim$ one per year per star at most. However the Swift BAT widefield monitor has detected hard X-ray outburst for several active stars including EV Lac (Osten et al. 2009) and CC Eri, and the active binaries II Peg (Osten et al. 2007) and Algol. At the peak of superflares the flare X-ray luminosity can exceed the stellar photospheric bolometric luminosity. During superflares a significant hard X-ray continuum is detected out to at least $50 \mathrm{keV}$; such high-energy photons can significantly alter grain and planetesimals structure through annealing and melting. These large flare outbursts provide a vast increase in the $\mathrm{X}$-ray radiation field with significant photon flux capable of penetrating deep into the dead zone of a protoplanetary disk.

\section{Conclusions and Future Possibilities}

The nearest examples of accreting PMS stars ("classical" T Tauri stars) are at distances of $\sim 150 \mathrm{pc}$ in the nearby star formation regions, such as Taurus-Auriga, Chamaeleon, Upper Scorpius etc.; hence these are difficult targets for even moderate resolution FUV and X-ray spectroscopy. The situation is more favorable for older post-accretion stars because many nearby, young, single active dwarfs are members of the Local Association (Eggen 1992), which is a widespread aggregate of young stars related to star formation in the Sco-Cen association. By chance the Sun lies within the Local Association, thus its members cover the whole sky and many are very near $(\leqslant 50 \mathrm{pc})$. Many of these stars were identified because of their strong coronal activity (Montes et al. 2001; Zuckerman \& Song 2004; Torres et al. 2008) The Local Association has been subdivided enthusiastically by many researchers, because, while Local Association ages span the range 7-300 Myr, the age spreads of the subgroups are much narrower. In our examples above we showed spectra of AU Mic, a particularly well-studied M1 dwarf member of the 12 Myr old $\beta$ Pic Moving Group, which at a distance of only 9.9 pc from the Sun shows an easily resolvable debris disk.

So what are we doing to better understand PMS star high-energy radiation fields? Full datasets presently exist for only a few stars but much more can be done now using existing space-based observatories. Current instrumentation can now measure the UV and X-ray spectra of many critical example stars being studied extensively in other spectral regions. The COS spectrograph (Froning \& Green 2009) on the HST is the ideal instrument to efficiently survey the FUV+NUV spectra of young stars. The COS medium resolution grating can measure both accretion diagnostics and the fluxes and profiles of more normal emission lines formed over a wide range of temperatures. Over the next year (2010) we shall be acquiring data for a carefully selected sample of young stars with accretion rates spanning 3-4 orders of magnitude and also probing stars with transitional disks and where dust disks have disappeared. [HST Large Program (GO11616; PI Herczeg) with 32 GO targets]. This sample is already well-studied in the infrared and X-ray spectral regions and detailed multi-wavelength modeling of these stars should provide significant insights into the evolution of PMS stars and their disks. 
I thank Rachel Osten for extensive discussions relating to X-ray superflares and for permission to quote her results ahead of publication. I thank Edmund Hodges-Kluck for his assistance in the reduction and analysis of the Chandra HETGS spectrum of AU Mic. This work was supported by Chandra grants GO8-9005X, GO9-0015X, and GO9-0020B, HST GO grant 11336, FUSE grants NNX07AU48G and NNX07AU51G, and NASA LTSA grant NAG5-13058.

\section{References}

Alexander, R. D., Clarke, C. J., \& Pringle, J. E. 2006, MNRAS, 369, 229

Bergin, E. A. 2009, in: P. Garcia (ed.), Physical Processes in Circumstellar Disks Around Young Stars, (Chicago: University of Chicago Press), in press

Blum, J. \& Wurm, G. 2008, ARAA, 46,21

Cully, S. L., Siegmund, O. H. W., Vedder, P. W., \& Vallerga, J. V. 1993, ApJ(Letters), 414, L49

Dullemond, C. P., Hollenbach, D., Kamp, I., \& D'Alessio, P. 2007, in: B. Reipurth, D. Jewitt, and K. Keil (eds.), Protostars and Planets $V$ (Tucson: University of Arizona Press), p. 555

Eggen, O. J. AJ, 104, 2141

Feigelson, E. \& Montmerle, T. 1999, ARAA, 37, 363

Froning, C. S. \& Green, J. C. 2009, ApधESS, 320, 181

Glassgold, A. E., Najita, J., \& J. Igea, J. 2004, ApJ, 615, 972

Gorti, U. \& Hollenbach, D. 2009, ApJ, 690, 1539

Güdel, M., Briggs, K. R., Arzner, K., Audard, M., Bouvier, J., Feigelson, E. D., Franciosini, E., Glauser, A., Grosso, N., Micela, G., Monin, J.-L., Montmerle, T., Padgett, D. L., Palla, F., Pillitteri, I., Rebull, L., Scelsi, L., Silva, B., Skinner, S. L., Stelzer, B., \& Telleschi, A. 2007, A\& A, 468, 379

Montes, D, López-Santiago, J., Gálvez, M. C., Fernández-Figueroa, M. J., De Castro, E., \& Cornide, M. 2001, MNRAS, 328, 45

Osten, R., Drake, S., Godet, O., Pye, J., Barthelmy, S. D., Cummings, J., Pal'shin, V., Golenetskii, S., Tueller, J., \& Krimm, H. 2008, ATel, 1499

Osten, R. A., Drake, S., Tueller, J., Cummings, .J, Perri, M., Moretti, A., \& Covino, S. 2007, ApJ, 654, 1052

Osten, R. A., Hawley, S. L., Allred, J. C., Johns-Krull, C. M., \& Roark, C. 2005, ApJ, 621, 398

Owen, J. E., Ercolano, B., Clarke, C. J., \& Alexander, R. D. 2009, MNRAS, in press

Stelzer, B., Flaccomio, E., Briggs, K., Micela, G., Scelsi, L., Audard, M., Pillitteri, I., \& Güdel, M. 2007, $A \& A, 468,463$

Torres, C. A. O., Quast, G. R., Melo, C. H. F., \& Sterzik, M. F. 2008, in: B. Reipurth (ed.) Handbook of Star Forming Regions Vol. II (San Francisco: Astronomical Society of the Pacific), p. 757

Wyatt, M. C. 2008, ARAA, 46, 339

Zuckerman, B. \& Song, I. 2004, ARAA, 42, 685 